\title{
Context-Aware Business Processes Modelling: Concepts, Issues and Framework
}

\author{
Khouloud Boukadi *. Amin Chaabane** \\ Lucien Vincent* \\ *École des Mines de Saint-Étienne \\ Division for Industrial Engineering and Computer Sciences \\ 158 cours Fauriel, F-42023 Saint Etienne Cedex 2, France \\ e-mail: \{boukadi,Vincent\}@emse.fr \\ **École de Technologie Supérieure \\ Department of Automated Manufacturing Engineering \\ 1100 Notre Dame Ouest, Montreal, Quebec, Canada, H3C $1 \mathrm{~K} 3$ \\ e-mail: amin.chaabane@etsmtl.ca
}

\begin{abstract}
Recent research activities on the design of business processes were driven by flexibility. Process flexibility can be achieved through the ability to trigger dynamic changes in the core of business process components in a timely manner and in response to business environment evolution. The drivers for business process flexibility can be found in the "Context" of a process which may include among others time, location, weather, legislation, and culture. Context-Aware Business Process Modelling extends traditional business process models with contextual variables that trigger a dynamic behavior under continuous change. However, as yet, most of the research work in this area has focused on some specific contextual information to model some varying parts such as business rules and goals. Little attention has given to the design and development of a general framework for context-aware business process modelling. In this paper, major business process components were extracted from the main existent business process design formalisms, amplified by contextual information, and integrated into a more a general framework. A case study of a well known European Logistics Enterprise is used to illustrate how contextual information should be integrated within existent business process modeling approaches.
\end{abstract}

Keywords: Business Process Modelling, Process Flexibility, Context-awareness.

\section{INTRODUCTION}

The development of information systems has evolved from a monolithic standalone style to a component-based approach. Now, applications are designed and developed from one or several services coordinated by a Business Process Model. This modern concept is known as Service Oriented Architecture (SOA). SOA is a promising architectural approach for designing, architecting and delivering loosely coupled integration where software capabilities are exposed as business-meaningful services. The successful use of SOA is based on its symbiotic relationship with the business process modelling (BPM) field. Indeed, as to SOA, business processes will be implemented on the basis of an individual composition of different services. However, the service modelling process oftentimes fails to capture various servicespecific concerns required for delivering context-aware and user-friendly business services. This is due to the fact that, business processes, considered as the essence of services identification, are not modelled to be aware to changes in the company's environment and customer's expectations. Business process flexibility is an important issue which must be taken into account when designing business processes under the SOA architecture. Business process flexibility can be defined as the capacity to implement changes in the business process types and instances by modifying elements such as activities, events and flows. The lack of flexibility leads to sub-optimal design of multiple business processes for different situations with high level of redundancy and significant maintenance. Despite the innovative research proposed by different authors, there is still a lack of an integrated methodology that supports variability according to the contextual information of process model for the enterprise. We believe that "contextual information" becomes an essential element that must be added in the design phase of a business process.

The remainder of this paper is organised as follows. Section 2 presents background and an overview about the ongoing related work on Context-aware Business Process Modelling (C-BPM). The third section shows the differing BPM techniques and provides an in depth comparison of five well known BPM techniques. Several main components are identified and categorised. This analysis demonstrates that all business process formalisms don't include the contextual information explicitly in the BPM phase. Section 4 focuses on the integration of contextual information into business process modelling and proposes a generic framework for context-aware business process design through a general taxonomy of this information. Therefore, a mapping of the structure of the different contextual information to business process component is presented. An illustrative case study is used to demonstrate the applicability of the framework and the value of such an approach. Finally, we conclude with a summary of the proposed work with a discussion about future research activities in the field of flexible (context-aware) business process modelling or design. 


\section{BACKGROUND AND RELATED WORK}

Business process flexibility is a well established area of research and continues to attract both practitioners and researchers. Gartner argue (Gartner, 2005) that flexibility will drive the emergence of business process management in the future because it will support enterprise competitive goals. Context-aware business modelling is relatively new field of research and holds much promise for a greater understanding of business process and increasing its flexibility. This concept has been studied in various fields for quite a long time. There are a number of different definitions and uses for this term. Context appears in many disciplines as a "metainformation" which characterizes the precise situation of an entity to describe a group of conceptual entities, or as a logical construct to facilitate reasoning services (Benslimane et al., 2006). Schilit and Theimer (1994) describe context as location, identities of nearby people, objects and changes in these objects. Dey et al. (2001) defines context as: "Any information that can be used to characterize the situation of an entity. An entity is a person, place or object that is considered relevant to the interaction between a user and an application, including the user and the application themselves".

The introduction of context awareness is not novel in information systems. Different related disciplines have already used the notion of context such as Web system engineering (Kaltz et al., 2005) and mobile applications (Mikalsen et al., 2004). Contextual information can vary from one area to another. In mobile applications, the aim is to provide users with ubiquitous access to information, communication and computation (Schilit et al., 1994). A typical example might be to find a nearby restaurant at which a particular kind of food is served. In this area, context categories are concerned with three entities: places (rooms, buildings, parking etc.), people (individuals or groups), and things (physical objects, software devices). Each of these entities may be described by various attributes which can be classified into four classes: identity (each entity has a unique identifier), location (entity's position, co-location, proximity, etc.), status (or activity, meaning the intrinsic properties of an entity, e.g., temperature and lightning for a room, etc.) and time (used for timestamps to accurately define situation, ordering events, etc.). In the field of Web services, context is used to facilitate the development and deployment of adaptable Web services (Maamar et al., 2008). Maamar et al. (2005) define the context as an aggregation of three categories: the user context (U-context), the Web service context (W-context) and the resource context (R-context). The U-context tracks the current status of users and reflects their personal preferences in terms of execution location and execution time of services. The W-Context includes information about the current status of a Web service and its execution constraints. The R-context monitors the current status of a resource needed to execute a service. In the field of the Web service based collaborative business process, Boukadi et al. (2008) propose a context categorization ontology in which context definition belongs to a certain category which can be provider, customer and collaboration related.
Context-aware business process modelling and design is a new area of interest. Rosemann and Recker (2005) define business process context as: "the minimum set of variables containing all relevant information that impact the design and execution of a business process". In addition, they propose a research agenda which discusses a set of research questions which concern the design of context-aware business process. This paper can be perceived as an excellent starting point which presents some guidance by providing fundamental research questions. Saidani et al. (2007) address the relevance of context related knowledge (CRK) for business process modelling. In their work, these authors discuss key issues related to the support of CRK including the elicitation of CRK, as well as its categorisation, adaptation, measure and use for business process instantiation. Besides, they introduce taxonomy for context which captures most common CRK. But, the proposed taxonomy is not broad enough to be applied in all sorts of business process. Moreover, how these contextual elements can be incorporated in the business processes design is not developed.

\section{REVIEW OF BUSINESS PROCESS MODELLING}

BPM is typically performed by business analysts in order to improve process efficiency and quality. In the following, five of the most common techniques (Aguilar-Savén, 2004) are analysed in order to determine the basic modelling components of business processes which will be used in creating a context-Aware Business Process Modelling framework. The Event-driven Process Chains (EPC) developed by Keller, Nuttgens and Scheer (1992) are based on Petri-Nets where activities and states are equal. At the heart of the EPC notation are functions which represent activities, events which represent the state of the process and connectors which split or join process flow. EPC can also indicate which organisational unit is responsible for each function and what information or objects are consumed and produced. Control flow such as AND, OR and XOR logic is supported by EPC and is represented by circular icons which may be placed between events and functions.

Role Activity Diagrams $(R A D)$ is another technique that was developed in 1987 by Praxis ${ }^{1}$ as a part of their "Systematic Technique for Role and Interaction Modelling" (STRIM) (Ould, 1995). RAD was designed mainly for software processes and contains five key concepts: roles, actors, activities, interactions and entities. Integrated computer-aided manufacturing DEFinition 0 (IDEF 0) is one among several techniques which is part of the IDEF methodology and designed by the US Air Force as a way to increase productivity in manufacturing with the aid of computer technology. Each IDEF technique $(0,1,2 \ldots)$ represents the system under investigation from a different perspective. For example IDEF0 provides a functional view of the system, IDEF1 models the information and IDEF3 simulates the behavioural perspective. IDEF0 depicts process through its functions by specifying how inputs (flows arriving from the left are transformed into outputs via controls and

\footnotetext{
${ }^{1}$ http://www.praxis-his.com
} 
mechanisms. Inputs and outputs are any entity that can be acted upon. IDEF3 relies on two diagram types to fully describe processes: the process flow and the object state transition diagrams. As seen with IDEF0, IDEF3 models support hierarchies.

The Unified Modelling Language (UML) is increasingly being seen as the de-facto standard for software modelling and design. UML Activity Diagrams (UML AD) are the most detailed form of process modelling within UML. In this type of diagram, the fundamental unit of behaviour specified is the activity. Activities are composed of actions and/or other activities and they define dependencies among their elements. Graphically, they are composed of nodes and edges. The edges connect the nodes in sequential order. Nodes represent actions, activities, data objects, or control nodes. Besides, UML AD propose a series of twenty common control-flow modelling requirements that occur when defining process models. Finally, the Business Process Modelling Notation (BPMN) is a technique which is may become the standard for the representation of business processes. BPMN development has focused on the ability to model workflow in the "Business Process Execution Language for Web Services" (BPEL4WS). Not surprisingly, UML Activity Diagrams and BPMN share many similarities. The main icons are activities, AND, OR, and XOR logic, swimlanes, starts, ends and subprocesses. Unlike UML AD, BPMN does not support object flow. BPMN can however model the information flow between activities which are called message flows.

The previous overview of BPM formalisms demonstrates that six basic components are considered for almost all modelling techniques: activities, events, flow controls, connections, artifacts, and swimlanes as shown in Fig. 1.

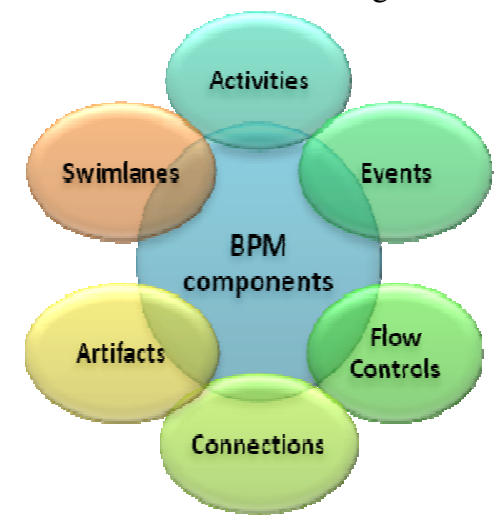

Fig. 1. Core set of BPM components

Indeed, the abstraction of a real-life process into a visual representation can be made by the concept of activities. All process modelling methodologies seek to describe a system by documenting the different activities that can occur. When process models contain many activities, it may become difficult to quickly identify the first and the last activities. Thus, the modeller must make a clear distinction of which activities have precedence and which are subsequent. The use of distinct symbols or icons to represent starts and ends in the model. Thus, an event is something that happens during the course of a business process. There are three types of events, based on when they affect the flow: start, intermediate, and end. While activities and events are the foundation of most modelling formalisms, almost as many methodologies have some concept of flow controls. This will determine branching, forking, merging, and joining of paths. Internal markers will indicate the type of behavioural control. Splits allow for the completion of one activity to branch into two or more subsequent activities rather than just one. In addition, connections are used to connect the flow between activities, events and flow controls which represent the flow objects. To design a detailed business process, modellers need to show some additional information (data, text, etc, ) about a process that is neither related to the sequence for flow of the process nor to the flow of message. Thus, artifacts are used as additional standardised information for business process modelling. And finally Swimlanes add another layer of information to business process models by drawing a distinction between the various actors (participants, roles or organisational units) who execute the activities of a process.

Most modelling techniques found in literature suppose that business processes are static and there is no need to change after development. However, the business environment is in continuous change and thus the need to integrate flexibility in business process design is paramount.

\section{FRAMEWORK FOR CONTEXT-AWARE BPM}

BPM is usually used to facilitate the execution and automation of different tasks, but does not use contextual information as shown earlier in the overview of the different methodologies. For a business process to be context-aware, we believe that it should be supported by a model that provides the means for categorising, naming, storing, retrieving, reasoning with and binding the contextual information to the core set of BPM elements. The categorisation of context is an important stage in the design of BPM because each category is parameterised by a specific set of characteristics. Figure 2 depicts our reference frame that is used to define and integrate the relevant contextual information. As aforementioned, three main categories can be used during the specification of contextual information related to business process: functional, non-functional and environmental contexts. The contextual variables differ from one category to another. In the following we introduce and discuss these categories.

The functional context includes information that is directly connected to the business process. It includes three subcategories: the role-based context, business rules, and the business goals. The role-based context is related to the sensitivity of the business process to roles (actors or resources). Contextual information about actors describes their most important characteristics such as profile, expertise (e.g. experience level) and other properties in relation with work (e.g. "motivation", "performance", "ego involvement", etc.). This information expresses a rich set of constraints that provide a more appropriate match so that only the pertinent actor which plays the role can perform a given activity. For resources, contextual information includes resource availability, cost, reliability, etc. The second sub-category is the business rule. This describes the instructions and business policies that govern business process activities. Finally, the 
third sub-category describes the contextual information about business process goal or goals that need to be fulfilled during process execution. These goals can be extracted form the business process motivation models. Therefore, the functional context is important because it helps to identify the business process constraints and thus business service constraints when going to a service based solution.

The second category, the non-functional context, captures elements that are not directly related to the process and in most cases; these elements are beyond the control of the business process itself. Although this non-functional contextual information does not influence the design of the business process, it still makes an impact on the way the business process is executed. Non-functional context variables can be the parameters for quality and security of the business process model.
The third category is the environmental context. It specifies particularly the environment in which the business process is embedded. It includes variables such as time (e.g. different business models depend on the time of day or the period of the year), location (e.g. separate business models for other countries), legislation (e.g. differing regulations or constraints among different regions) and culture. Each of these variables is clearly associated with a comprehensive set of changes in the business process. At this level, the proposed categorization is dynamic in the sense that new subcategories may be added at any time. Besides, between categories many relationships may occur (e.g., a new business rule is created in response to new legislation"). In the same category, sub-categories may also influence other sub-categories (e.g., if a resource assigned to a particular business activity is overloaded, a business rule can suggest that an actor choose an alternative resource).
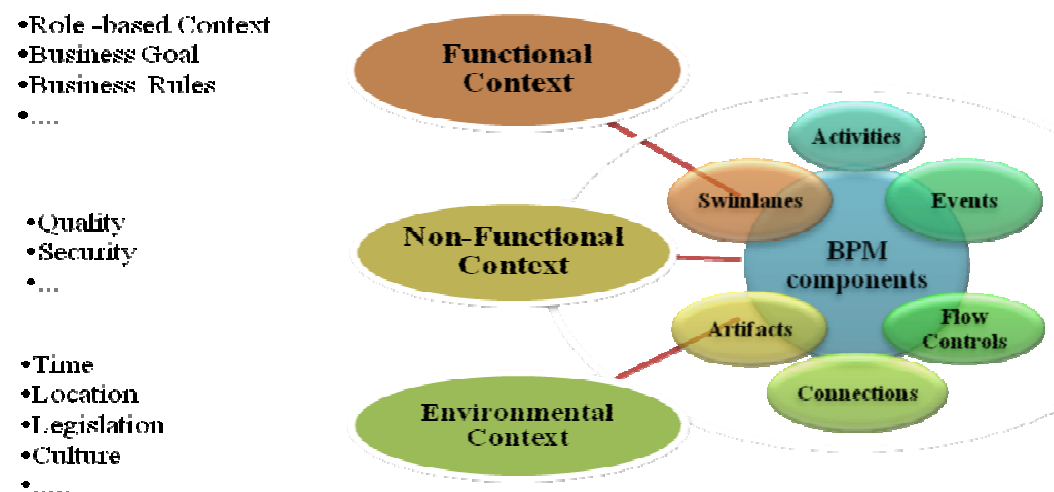

Fig. 2. Context-Aware Business Process Modelling Framework

Using this framework, it is easier to understand different contextual information and their impact on each business process component. In the following, we give a brief indication of their integration within activities, events, control and flow context.

Activity-related context includes the three categories. The functional context contains three elements: the role that should process the activity, business goal, and business rule. The non-functional context level covers elements such as security and quality of a given activity within a process. Finally, the environmental contexts of activities deal with elements such as the change of a business activity from one country to another due to the change of regulation. Eventrelated context consists in considering process information from different sources in order to respond better to various scenarios. In the event-related context, we identify two subcategories: functional and non-functional contexts. The functional context copes with business rules that constraint events. An example of such a rule constraining a start event is $<$ if customer demand does not exceed a certain threshold, don't trigger the execution of the business process $>$. The nonfunctional context reflects the features related to the security and quality attributes. Some events are sensitive to security concerns (e.g. "only certain actors are authorized to trigger a business process execution"). Other events could be quality sensitive (e.g. "the business process execution should not exceed two days"). Flow control-related context includes the functional context that could affect the flow controls among business activities. Business rule could be considered as the main constraining factor on flow control design. In this case, business rule expresses a guard condition, which is a semantic condition or restriction, that contains all the information required to make the decision whether to execute the flow control or not. Finally, artifact-related context can represent a set of information to characterize data objects exchanged among business activities. This information is captured in the functional level which includes only the role based context. For example, it can describe whatever an actor and/or a resource are involved in manipulating a data object.

\section{CASE STUDY}

In this section, we use a case study of a very well know European Logistics enterprise to illustrate the applicability of the proposed framework. This example demonstrates how the modeller can use the framework to redesign context-aware business processes. The delivery process is triggered when the delivery manager receives a "Delivery order" including the customer related information (e.g. customer location, target due date, etc.) and some other information about the merchandise to ship (e.g. quantity, nature, due date, etc.). Once employees are assigned to a delivery truck and a 
delivery is planned to the customer, the activity, "Picking merchandise from the warehouse", is initiated. For security reason, the employee should verify the merchandise before the shipping process. Verification tasks include quantities and goods nature controls. Once verified, "Putting merchandise in parcels" activity is executed and followed by a delivery price computation. Finally, the merchandise is shipped to the business opportunity location with respect to the delivery due date. A delivery confirmation message is sent to both customer and delivery manager. Figure 3 depicts the major components of the delivery business process model using BPMN formalism.

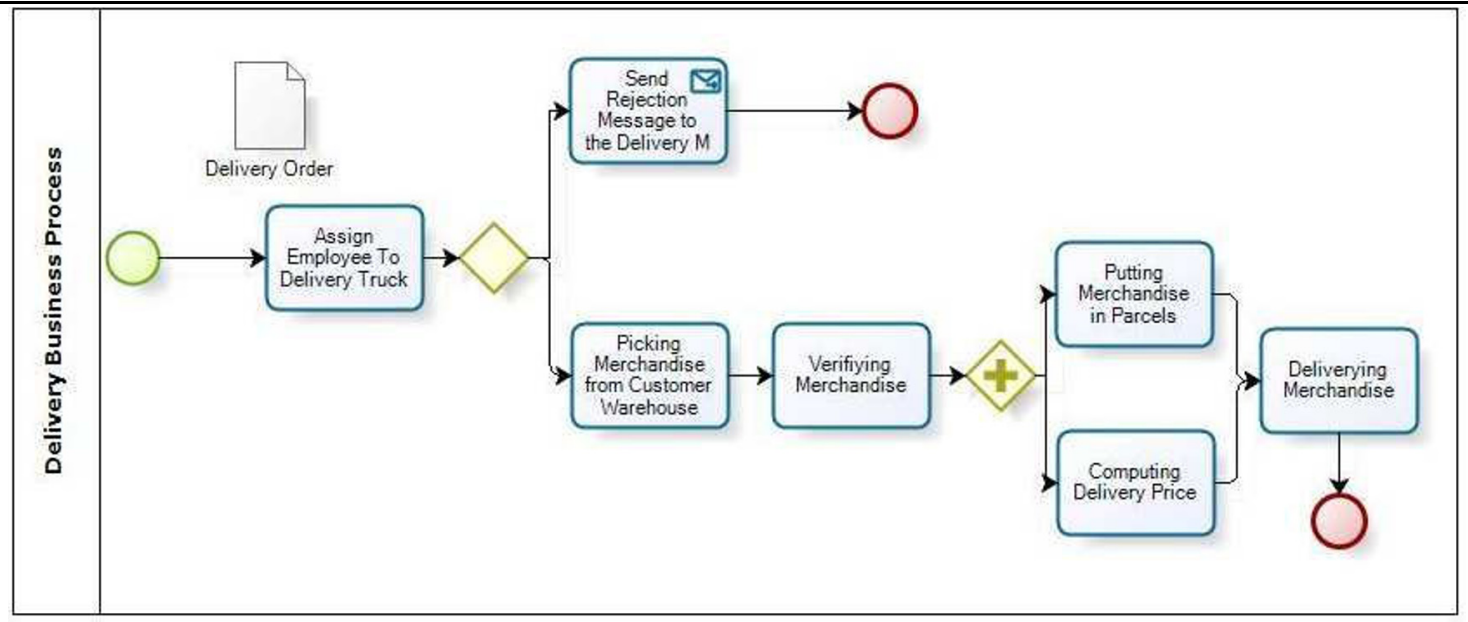

Fig. 3. Delivery Business Process

The delivery process typically runs smoothly in regular business circumstances. However, some parts of the process are subject to changes in response to different contextual information. For example, if the client is located outside the country, some specific controls for security reasons should be added or the price calculation procedure is different. Thus, the business process model should be amplified by external contextual information that trigger changes in the business process components (activities, flows, etc.) in a timely manner without a complete modification or redesign. In this case, environmental context, such as weather conditions (e.g. storm, flood, etc.) may affect the activity "putting merchandise in parcels". Consequently, several adaptation actions should be anticipated to avoid the deterioration of the merchandise. For example, metal boxes should be used instead of cardboard boxes. Moreover, the environmental context can have an impact on locations. While location as a contextual variable is widely discussed as part of research related to mobile applications, it has wider implications for process management. In this case study, the customer location seems to be very important (i.e., the delivery process may have different behaviours according to the current customer location). Information about the country where the process occurs is so relevant. Consequently, new considerations should be added to the process. For example, some flow controls should be added during the process execution. These controls are relative to the respective legislative regulations of the country. Hence, several scenarios lead to different safety levels that in turn require some specific procedures. As an example, employees should use more sophisticated alarming systems to verify the merchandise or they should run a test for explosive goods, etc. Fig. 4 shows particularly how the business process will change dynamically in order to be adjusted automatically. The change triggers two major modifications. The first one is at the flow-control level where a test on the delivery location is performed. The second change is at the activity level where two new activities are added, when compared with the standard delivery process. The new activities are "Verifying merchandise using alarming system" and "Testing for explosive". In addition, some countries may introduce quota to restrict the volume of transported merchandise. In this case, the delivery process should assign several delivery trucks in order to avoid customs duties. The time related information is another environmental contextual variable that can impact the delivery business process. As usually known, the volume and the delivery frequency may be significant depending on the period of the year. During the Christmas period, the quantities to ship are usually important. The related process changes can be anticipated simply by regularly observing the demand forecast through artifactsrelated contextual information, which in turn enables a best planning of resources (number of trucks and employees). The delivery process can have also a functional related context such as business rules which are context-dependant rules. Assume that a business rule $\mathrm{R} 1$ is aware of the past interactions with customers (historical relationships) and impacts the verification merchandise activity. The past interactions with customers may be either "past interaction=No" or "past interaction=Yes". In the first case, the verifying merchandise business activity is performed, but skipped in the second case. Finally, an example of nonfunctional contextual information consists of integrating the financial situation of the customer when the delivery process is initiated. Thus, if the customer has some financial problem, a further investigation is initiated and a sub-process is added. The previous case study demonstrates that the proposed framework is not only able to classify contextual information but also trigger the change needed in each business process component. The main advantage of capturing context information externally form to the business process model is potentially to build a library of contextual parameters. The 
maintenance and extension tasks are much easier as opposed to context information that is buried within various business process models. Now, the challenge consists of analyzing different types of contextual influences and determines their consequences to each component of the traditional business process.

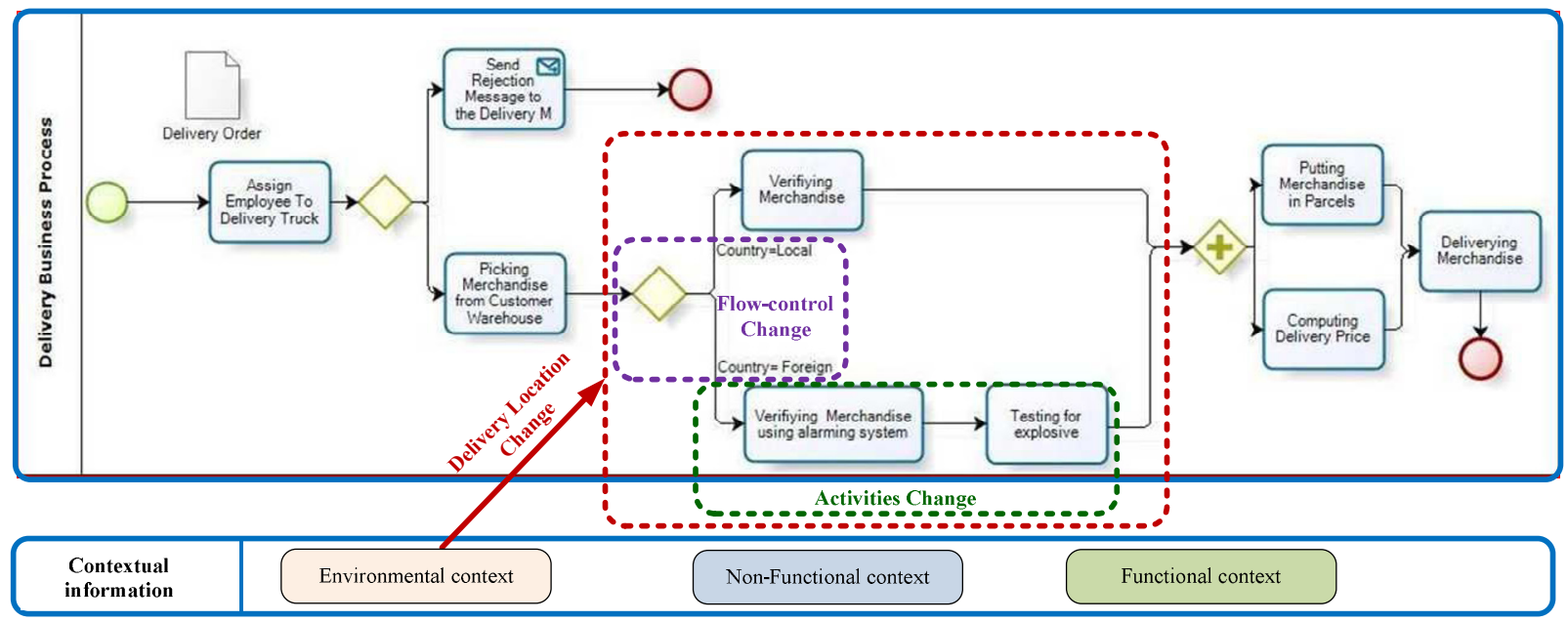

Fig. 4. Delivery Business Process with context consideration

\section{CONCLUSIONS}

This research highlights the relevance of contextual information for business process modelling to increase process flexibility. A detailed analysis of existent BPM methodologies demonstrates that six basic components are important for modelling business processes which are activities, events, flow controls, connections, artifacts, and swimlanes. Although business process modelling has attained a certain level of sophistication and can handle multiple elements, contextual information is still ambiguous. In this paper, we proposed a generic framework that presents an appropriate description and conceptualisation of business process contextual variables. These variables can be integrated as extension to existing process modelling formalisms and will be subject to future development. In addition, the proposed framework is subject to different improvements. Most importantly, an overall process management approach that aligns contextual variables with business process and drive business process flexibility through adaptive process management system is highly valuable.

\section{REFERENCES}

Aguilar-Saven R.S. (2004) "Business Process Modelling: Review and Framework", International Journal of Production Economics, Vol. 90, pp 129-149 (2004);

Benslimane, D., Arara, A., Falquet, G., Maamar, Z., Thiran, P., Gargouri, F. (2006). Contextual Ontologies: Motivations, Challenges, and Solutions. Proceedings of the Fourth Biennial International Conference on Advances in Information Systems, Springer (ED), Izmir, Turkey, pp. 168-176.

Boukadi, K., Ghedira, C., Vincent, L. (2008). An Aspect Oriented Approach for Context-Aware Service Domain Adapted to EBusiness. Proceedings of the 20th International Conference on Advanced Information Systems Engineering (CAiSE'08), Montpelier, France, pp.64-78.

Dey,A. K., Abowd, G.D., Salber, D.(2001). A conceptual framework and a toolkit for supporting the rapid prototyping of context-aware applications. Human-Computer Interaction, 16 (2), pp. 97-166.

Gartner (2005). Flexibility Drives the Emergence of the Business Process Platform (Publication number. G00126854). Stamford, CT: Y. Genovese, S. Hayward, G. Phifer, D. C. Plummer, J. Comport, D. M. Smith.

Kaltz, J.W., Ziegler, J., Lohmann, S. (2005). Context-aware Web Engineering: Modeling and Applications. Revue d'Intelligence Artificielle, 19 (3), pp.439-458.

Keller, G., Ntlttgens, M., Scheer, A.W. (1992), "Semantische Prozebmodellierung auf der GrOndlage 'Ereignisgesteuerter ProzeBketten (EPK)"', Institut fur Wirtschaftsinformatik, Paper 89, Saarbrucken.

Maamar Zakaria, Mostefaoui Kouadri Soraya, Mahmoud Qusay H. (2005) Context for Personalized Web Services. Proceedings of the 38th Annual Hawaii International Conference on System Sciences (HICSS'05), (07), pp. 166-171.

Maamar, Z., Sheng, Q.Z., Benslimane, D., Thiran , P. (2008). Web Services, Policies, and Context: Concepts and Solutions, Advances of Computational Intelligence in Industrial Systems, 116, pp. 39-55.

Mikalsen, M., Kofod-Petersen, A. (2004). Representing and Reasoning about Context in a Mobile Environment. Proceedings of the First International Workshop on Modeling and Retrieval of Context, Germany, pp.25-35.

Ould, M.A. (1995) Business Processes: Modelling and Analysis for Re-Engineering and Improvement. John Wiley \& Sons.

Rosemann, M., Recker, J. (2006). Context-aware Process Design Exploring the Extrinsic Drivers for Process Flexibility, In proceedings of the18th International Conference on Advanced Information Systems Engineering., Luxembourg.

Saidani Oumaima, Nurcan Selmin (2007). Towards Context Aware Business Process Modelling. Proceedings the 8th Workshop on Business Process Modelling, Development, and Support (BPMDS'07), June 11-12, 2007, Trondheim, Norway.

Schilit, B., Adams, N., Want, R. (1994). Context-Aware Computing Applications. Proceedings of the IEEE Workshop on Mobile Computing Systems and Applications, December 8-9.

Schilit, B., Theimer, M. (1994) "Disseminating Active Map Information to Mobile Hosts". IEEE Network, 8(5).

Workflow Management Coalition, "The Workflow Reference Model", January 1995, retrieved from: http://www.wfmc.org/standards/standards.htm. 